\title{
Expression profiles of differentially expressed genes affecting fecundity in goat ovarian tissues
}

\author{
Y.H. Ling ${ }^{1,2 *}$, Q. Quan ${ }^{1,3 *}$, H. Xiang ${ }^{1,2}$, L. Zhu ${ }^{1,2}$, M.X. Chu ${ }^{4}$, X.R. Zhang ${ }^{1,2}$ and \\ C.Y. $\operatorname{Han}^{1,2}$ \\ ${ }^{1}$ College of Animal Science and Technology, Anhui Agricultural University, \\ Hefei, Anhui, China \\ 'Local Animal Genetic Resources Conservation and Biobreeding Laboratory of \\ Anhui Province, Hefei, Anhui, China \\ ${ }^{3}$ College of Economy and Technology, Anhui Agricultural University, Hefei, Anhui, \\ China \\ ${ }^{4}$ Key Laboratory of Farm Animal Genetic Resources and Germplasm Innovation of \\ Ministry of Agriculture, Anhui, Beijing, China \\ ${ }^{*}$ These authors contributed equally to this study. \\ Corresponding author: C.Y. Han \\ E-mail: hancy789@163.com
}

Genet. Mol. Res. 14 (4): 18743-18752 (2015)

Received July 28, 2015

Accepted October 25, 2015

Published December 28, 2015

DOI http://dx.doi.org/10.4238/2015.December.28.23

ABSTRACT. Although RNA-Seq is an effective method for identifying and exploring novel functional genes in mammals, it has rarely been applied to study fertility-related genes in the goat. In this study, RNA-Seq was used to screen the estrus ovaries of uniparous and multiparous Anhui white goats (AWGs). In total, 15,890 genes were identified and 2201 of these were found to be differentially expressed between the genetic libraries from uniparous and multiparous goats. Compared to the uniparous library, 1583 genes were upregulated and 618 genes were down-regulated in the multiparous library. The FER1L4 gene showed the level of highest up-regulation in the multiparous library, while SRD5A2 expression showed the greatest down-regulation. 
In order to determine the functions of FER1L4 and SRD5A2 in goats, the expression profiles of the two genes in different tissues from AWGs and Boer goats at diestrus were analyzed by quantitative PCR. FER1L4 and SRD5A2 showed tissue specific expression patterns and were highly expressed in ovaries from both AWGs and Boer goats. FER1L4 was more highly expressed in ovaries from multiparous than uniparous AWGs. In contrast, SRD5A2 was expressed at a lower level in multiparous AWGs. These results indicated that FER1L4 and SRD5A2 may be associated with the high fecundity of AWGs.

Key words: Goat; Ovary; Fecundity; Differentially expressed genes; Tissue expression profile

\section{INTRODUCTION}

Litter size is one of the most important economic traits in goat production and varies among individuals and breeds. Fecundity has a direct impact on goat production efficiency and is a result of interactions among multiple genes and the environment. There has been some progress in characterizing the major genes involved in animal fecundity, such as identification of BMPR-IB (Mulsant et al., 2001; Davis et al., 2002), BMP15 (Galloway et al., 2000; Zhang et al., 2009), GDF9 (Hanrahan et al., 2004), and PRLR (Kmieć et al., 2006). However, information on the identity and roles of fertility-related genes is still relatively limited in the goat. In general, litter sizes are low in goats with one or two kids being normal (Sun et al., 2010). The increasing demand for goat meat has contributed to the rapid development of the goat industry in recent years, and improvements to breeding methods have become increasingly important.

The Anhui white goat (AWG) shows precocious puberty compared to other goat breeds, has relatively high fertility, and the skin can be used to produce high quality leather goods. AWG ewes are fertile all year round and can produce kids twice in one year or three times in two years. The AWG breed produces a relatively high number of kids, with average production of 2.3 lambs per litter. This breed is therefore an ideal model for studying goat breeding traits. Here, we have concentrated on the ovary as this has a very significant influence on the kidding rate.

The RNA-Seq method is now widely used to investigate the transcriptome as it allows both mapping and quantification of expressed sequences. For example, it has been used to detect differentially expressed genes in mice (Mortazavi et al., 2008), cattle (Huang and Khatib, 2010), humans (Hackett et al., 2012), and pigs (Gunawan et al., 2013). However, relatively little study has been made of fecundity-related genes in goat. In this study, we screened for differentially expressed genes between ovaries from uniparous and multiparous AWGs. This analysis identified FER1L4 and SRD5A2 as the two genes showing the highest level of differential expression. The expression profiles of these genes were compared in different tissues from AWGs in estrus and Boer goats in diestrus by real-time PCR to investigate their function. This study provides a molecular basis for new breeding approaches in this commercially important animal species.

\section{MATERIAL AND METHODS}

\section{Experimental materials}

The AWGs and Boer goats used in this study were obtained from Boda Livestock 
Technology Development Co., Ltd. of Hefei (Anhui, China). Six goats in estrus, including three uniparous and three multiparous AWGs, and three Boer goats in diestrus were selected randomly based on their breeding records in the previous five years. All experimental animals were of similar age and appearance. The animals were housed under the same conditions and fed the same diets until slaughter. After slaughter, the ovaries, heart, live, spleen , lung, kidney, uterus, oviduct, muscle (Longissimus), and intestine were obtained and immediately snap-frozen in liquid nitrogen, and stored at $-80^{\circ} \mathrm{C}$ until total RNA extraction. All the experimental procedures with AWGs in the present study were given prior approval by the ethics committee of Anhui Agricultural University, Anhui, China, under permit No. AHAU20101025.

\section{Main reagents}

We used the following kits: RNAiso Plus tissue extraction kit (Takara, Japan); Prime Script ${ }^{\mathrm{TM}}$ RT reagent kit with gDNA Eraser for reverse transcription: (Takara); FastStart Universal SYBR Green Master kit (ROX) for quantitative fluorescence analysis: (Roche, USA). We also used chloroform, isopropanol, and absolute ethyl alcohol from Wuxi Zhanwang Chemical Reagent Co. Ltd, China.

\section{Library preparation and sequencing}

Ovaries of three groups of goats, namely uniparous AWG, multiparous AWG, and Boer goat, were each ground to powder in liquid nitrogen. Total RNA was extracted from each sample using TRIzol (Takara) according to the manufacturer instruction. Equimolar quantities of RNA from each sample were combined into one pool.

Total RNAs from all samples was pooled prior to library preparation. The mRNA was first extracted from the total RNA using oligo (dT) magnetic beads and sheared into short fragments of about $200 \mathrm{bp}$. These fragmented mRNAs were then used as templates for cDNA synthesis. The cDNAs were PCR amplified to complete the library. An Agilent 2100 Bioanalyzer and ABI StepOnePlus Real-Time PCR System were used to analyze the sample library. The cDNA library was sequenced using an Illumina HiSeq ${ }^{\mathrm{TM}} 2000$ platform.

\section{Sequence analysis}

The quality of the raw sequence reads was checked and contaminating reads were removed, for example, low quality reads, adaptor reads, reads with $5^{\prime}$ primer contaminants, reads without a 3' primer, reads without the insert tag, reads with poly $(A)$, and reads shorter than $18 \mathrm{nt}$. The clean reads were mapped to goat reference gene sequences (http://goat.kiz.ac.cn/GGD/download. $\mathrm{htm}$ ) using SOAPaligner/SOAP2 (Li et al., 2009), allowing up to two base mismatches. The gene expression level was then calculated using the Reads Per Kilobase per Million reads (RPKM) method (Mortazavi et al., 2008). The statistical significance of the differential expression of each gene was determined using the P-value (Benjamini et al., 2001). The FDR (false discovery rate) $\leq 0.001$ and $\mid \log 2$ Ratio| $\geq 1$ were used as the threshold to identify differentially expressed genes.

\section{Real-time PCR}

cDNA was extracted using Prime ScriptTM RT reagent Kit with gDNA Eraser (Takara) 
according to the manufacturer instruction. The real-time (q-)PCR was carried out using an $A B I$ StepOnePlus ${ }^{\mathrm{TM}}$ Real-Time PCR System (ABI, USA) with a SYBR Green qPCR Mix to examine the expression profile of FER1L4 and SRD5A2 genes in different tissues. $\beta$-Actin was used as the internal control. The gene primers are listed in Table 1. The reaction mixture contained $7.5 \mu \mathrm{L}$ Rox,

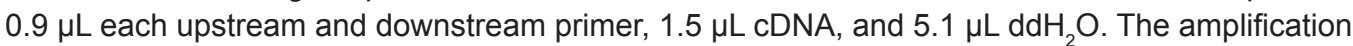
conditions were an initial denaturation step at $95^{\circ} \mathrm{C}$ for $10 \mathrm{~min}$, followed by $95^{\circ} \mathrm{C}$ for $15 \mathrm{~min}$, and 40 cycles at $60^{\circ} \mathrm{C}$ for $10 \mathrm{~min}$. All reactions were performed in triplicate. The melting curve of each gene was measured after amplification using SYBR Green with a fluorescein setting. Relative quantification was estimated using the comparative CT method, and relative gene expression levels were calculated using the $2^{-\Delta \Delta C t}$ method (Ishida-Takagishi et al., 2012).

\begin{tabular}{ll}
\multicolumn{2}{l}{ Table 1. Primer sequences used to amplify target genes. } \\
\hline Gene name & Primer sequences \\
\hline FER1L4 & F: 5'-CCGGCCTGGATACCTCTCTAT-3' \\
SRD5A2 & R: 5'-CAGTCGGCCTTGGAACAAA-3' \\
-actin & F: 5'-GCTCAGGAAGCCTGGAGAAAT-3' \\
& R: 5'-GGCATAGCCGATCCATTCAA-3' \\
\hline
\end{tabular}

\section{RESULTS}

\section{Analysis of RNA-Seq libraries}

Two RNA-Seq libraries were constructed from uniparous and multiparous samples and each generated over 5.9 million raw reads that were sufficient for the quantitative analysis of gene expression. After filtering out adaptor sequences, i.e., regions containing $\mathrm{N}$ sequences, and low quality sequences, each RNA-Seq library contained over 5.8 million clean reads; the uniparous and multiparous libraries gave 98.60 and $98.16 \%$ clean reads, respectively (Figure 1), demonstrating that both libraries were high-quality.

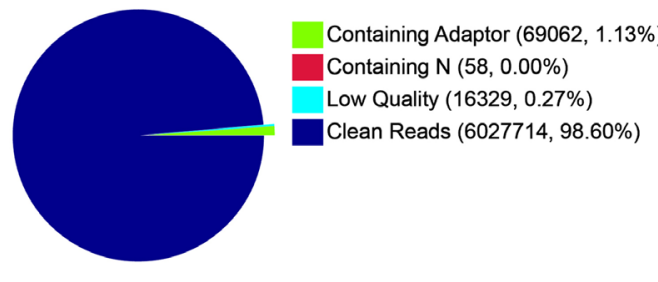

Composition of Raw Reads (Uniparous)

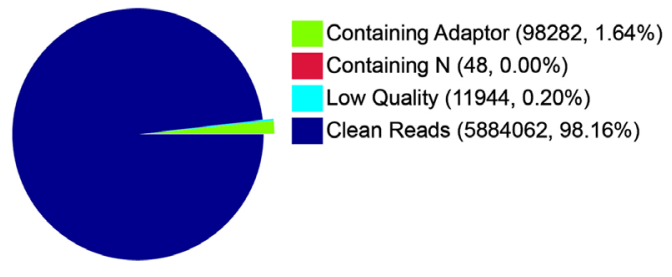

Composition of Raw Reads (Multiparous)

Figure 1. Composition of total raw reads from the uniparous and multiparous libraries.

\section{Screening differentially expressed genes}

In total, 15,890 genes were detected and 2201 genes showed differential expression between the uniparous and multiparous libraries. Compared to the uniparous library, 1583 genes 
were up-regulated and 618 genes were down-regulated in the multiparous library with an FDR $\leq 0.001$ and a |log2Ratio| $\geq 1$ as standard (Figure 2). Of these differentially expressed genes, FER1L4 showed the greatest up-regulation and SRD5A2 the greatest down-regulation in the multiparous library. The 10 genes showing the largest differential expression effects are listed in Table 2.

Table 2. Ten genes showing the highest levels of differential expression.

\begin{tabular}{lcllc}
\hline Gene & log2 ratio (multiparous/uniparous) & P value & FDR & Up/down-regulation (multiparous/uniparous) \\
\hline FER1L4 & 10.49393799 & $2.24 \mathrm{E}-47$ & $1.94 \mathrm{E}-45$ & Up \\
GVINP1 & 10.47220442 & $1.50 \mathrm{E}-08$ & $1.42 \mathrm{E}-07$ & Up \\
GVIN1 & 10.26986667 & $1.20 \mathrm{E}-07$ & $1.00 \mathrm{E}-06$ & Up \\
EVI2A & 9.914612892 & 0.000122 & 0.000596714 & Up \\
EYA2 & 9.489224544 & $4.78 \mathrm{E}-07$ & $3.66 \mathrm{E}-06$ & Up \\
SRD5A2 & -14.22914539 & $3.42 \mathrm{E}-74$ & $5.54 \mathrm{E}-72$ & Down \\
SST & -13.25260436 & $2.15 \mathrm{E}-19$ & $5.65 \mathrm{E}-18$ & Down \\
IBSP & -13.20576698 & $2.68 \mathrm{E}-48$ & $2.43 \mathrm{E}-46$ & Down \\
BTN1A1 & -13.1823193 & $6.07 \mathrm{E}-33$ & $3.30 \mathrm{E}-31$ & Down \\
CSRP3 & -12.1761523 & $1.76 \mathrm{E}-15$ & $3.41 \mathrm{E}-14$ & Down \\
\hline
\end{tabular}

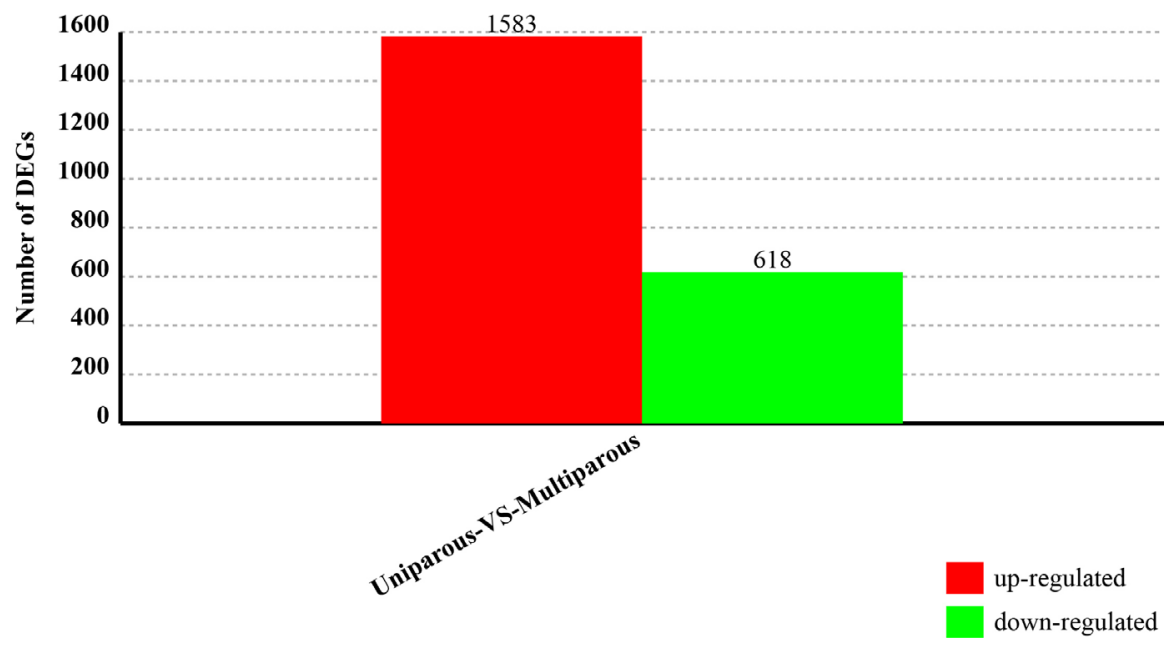

Figure 2. The number of differentially expressed genes between uniparous and multiparous samples. The red represents upregulated genes in the multiparous library, green represents genes downregulated in the multiparous library.

\section{Expression profile of FER1L4 in different tissues from AWG}

The expression profiles of FER1L4 and SRD5A2 were analyzed in different tissues of AWGs and Boer goats by q-PCR. The FER1L4 gene showed obvious tissue specificity (Figure 3). In uniparous AWGs, FER1L4 was most highly expressed in the small intestine, followed by kidney, spleen, lung, muscle, and ovary, but expression in the heart, liver, uterus, and oviduct was at very low levels. In multiparous AWGs, FER1L4 was most highly expressed in the ovary, followed by lung, but was expressed at very low levels in other tissues. The level of expression of FER1L4 was higher in the ovary of multiparous AWGs than uniparous animals. 


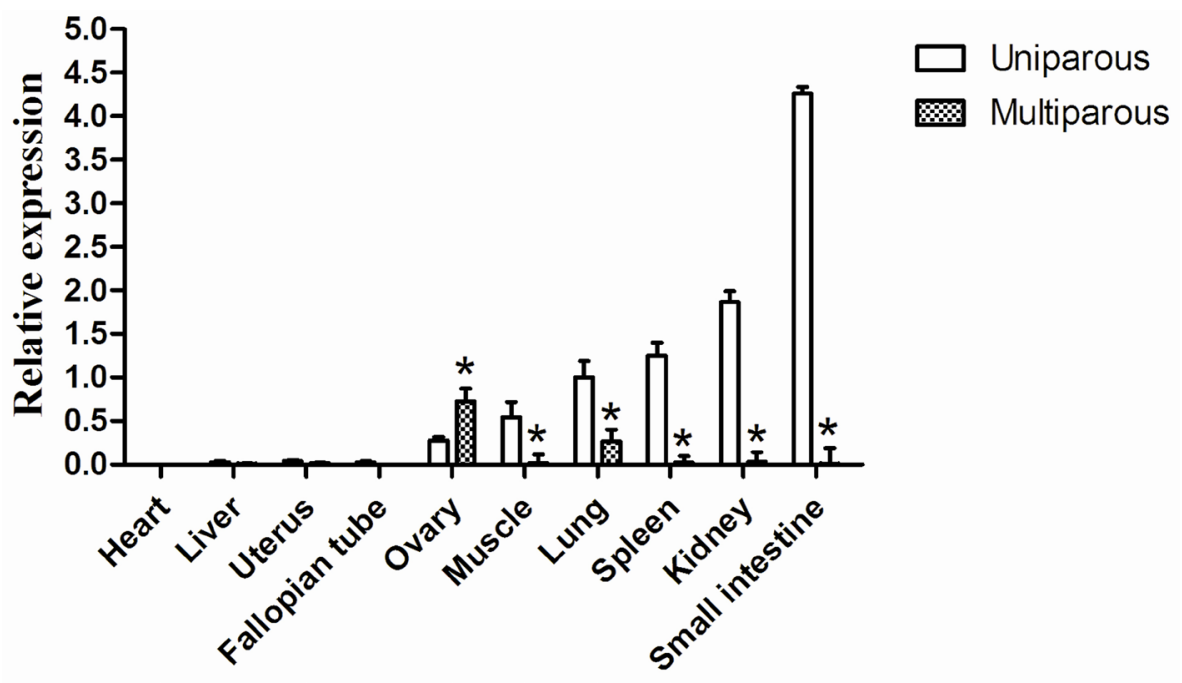

Figure 3. Expression profile of FER1L4 in different tissues of uniparous and multiparous Anhui White Goat. Asterisk: $P \leq 0.05$.

\section{Expression profile of SRD5A2 in different tissues from AWG}

Expression of SRD5A2 showed clear tissue specificity (Figure 4). In uniparous AWGs, $S R D 5 A 2$ was highly expressed in the liver, followed by kidney, muscle, and ovary, and showed lowest expression in the uterus and oviduct, with effectively no expression in other tissues. In multiparous AWGs, SRD5A2 was most highly expressed in the uterus, followed by small intestine and ovary, but showed little evidence of expression in other tissues. The level of SRD5A2 expression was lower in the ovary of multiparous AWGs than in uniparous animals.

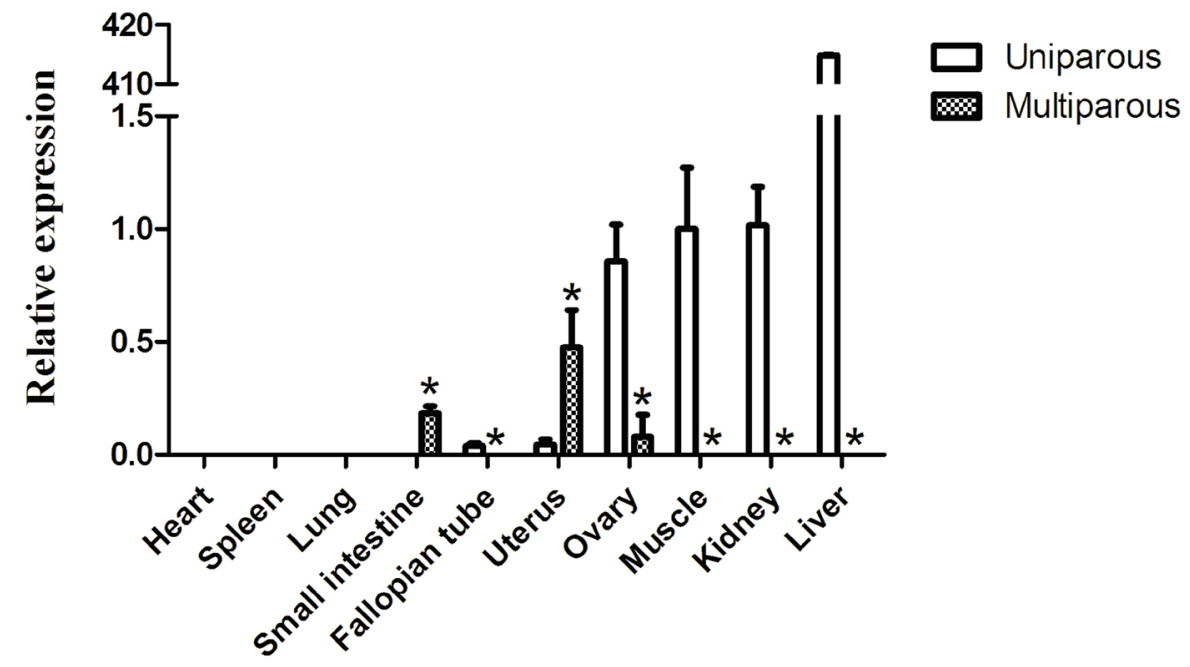

Figure 4. Expression profile of SRD5A2 in different tissues of uniparous and multiparous Anhui White Goats. Asterisk: $P \leq 0.05$. 


\section{Expression profile of FER1L4 in different tissues from Boer Goat}

FER1L4 showed clear tissue specificity in expression in different tissues from Boer goats (Figure 5). The highest level of expression was found in the oviduct, followed by spleen, small intestine, liver, ovary, and muscle, with little evidence of expression in other tissues. Boer goats showed higher FER1L4 expression in the ovary compared to AWGs.

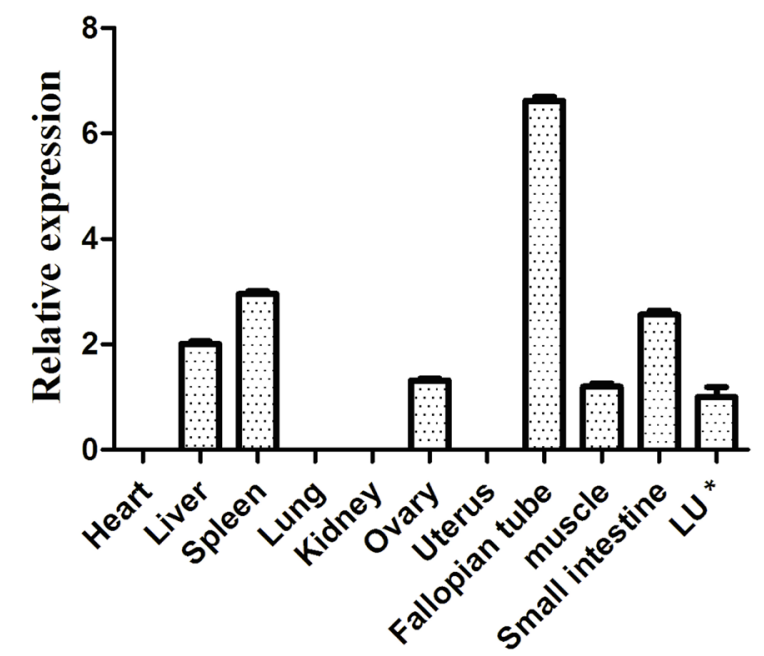

Figure 5. Expression profile of FER1L4 in different tissues of Boer goats. Asterisk: Ovary of uniparous Anhui White Goat.

\section{Expression profile of SRD5A2 in different tissues from Boer goats}

SRD5A2 also showed clear tissue specificity in Boer goats (Figure 6). Expression of SRD5A2 was highest in liver, followed by spleen, ovary, and muscle, but expression was difficult to detect in other tissues. Expression of SRD5A2 was higher in the ovary of Boer goats compared to AWGs.

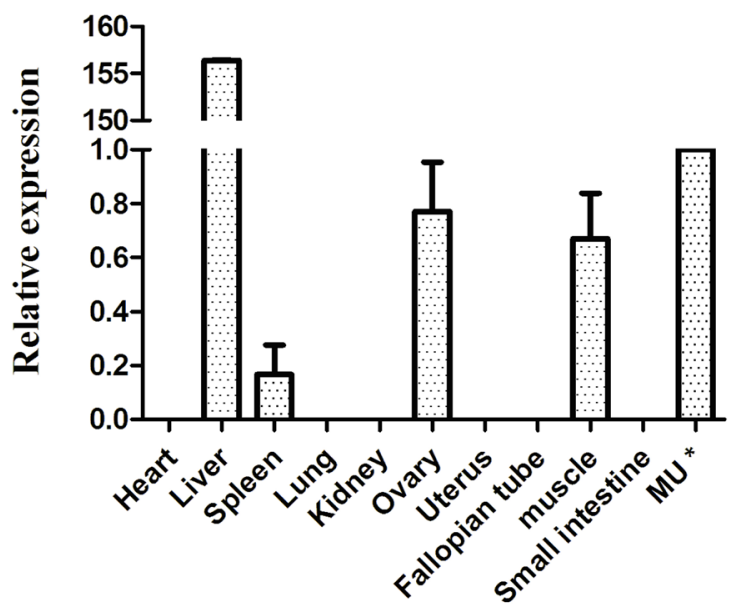

Figure 6. Expression profile of SRD5A2 in different tissues of Boer goats. Asterisk: Ovary of uniparous Anhui White Goat. 


\section{DISCUSSION}

\section{Screening and analyzing of differentially expressed genes}

In total, 2201 genes showed differential expression between uniparous and multiparous AWGs; this is a higher number than found in similar studies elsewhere (Gao et al., 2013). Although other studies have provided reference genomes of cattle and other close relatives of the domestic goat (Geng et al., 2013), these are of limited value to goat breeders due to species differences. However, the recently available goat genome sequence (published online 23 December 2012) has enabled the current analysis to be performed directly in the goat (Dong et al., 2013). The results here showed that more than $80 \%$ of the reads could mapped to the reference goat genome and the number of unmapped reads was significantly decreased. The effectiveness and accuracy of these results have increased dramatically and offer new information related to gene expression profiles of uniparous and multiparous goats. This data will provide more accurate and reliable information for follow-up studies.

\section{Function of FER1L4 and SRD5A2}

Currently, the function of FER1L4 is unknown. This gene is a recently discovered LncRNA and belongs to the FER1-like protein family (Song et al., 2013). An expression study showed that FER1L4 was significantly down-regulated in gastric cancer tissues (Song et al., 2013). The available evidence suggests FER1L4 might play a crucial role in human gastric cancer and be a new biomarker for clinical prognosis (Liu et al., 2014). However, the FER1L4 gene has been shown to be located in the cell membrane in the cellular component of GO categories. This suggests that FER1L4 may be involved in cell internal and external information exchange mechanisms. We found a high level of FER1L4 expression here suggesting that this gene may be a key candidate gene associated with high fertility in the goat.

Steroid 5a-reductase (SRD5A), including type-1 and type-2 5a-reductase, catalyzes testosterone ( $\mathrm{T}$ ) to dihydrotestosterone (DHT), and plays an important role in sex differentiation and androgen-mediated physiological processes. SRD5A2 is located on chromosome 2 p23 and has five exons and four introns. It is mainly expressed in the reproductive system and prostate tissue (Ellsworth et al., 1995), has a high affinity for steroid substrates, and participates in the reproductive system and prostate tissue development (Yokoi et al., 1998). SRD5A2 is also closely related with fertilization (Kang et al., 2014), sexual abnormality (Kostyrko et al., 1994), prostate disease (Kaefer et al., 1996; Akalu et al., 1999), polycystic ovary syndrome (Goodarzi et al., 2006), and cancer (Akalu et al., 1999; Li et al., 2013). Studies of human SRD5A2 deficiency pedigrees showed that male patients displayed pseudo-hermaphroditism, while female patients had reduced fertility (Hochberg et al., 1996). These characteristics are the opposite of the results in the present study. This discrepancy may be due to differences in post-transcriptional translation or species differences.

\section{Relationship between litter size and FER1L4 and SRD5A2 in AWGs}

We found that FER1L4 and SRD5A2 were highly expressed in the ovaries of estrus AWGs, and that the level of expression of FER1L4 was higher in multiparous than uniparous ovaries. $S R D 5 A 2$ behaved in a contrasting manner and was expressed at a lower level. 
Expression of FER1L4 and SRD5A2 was at high levels in the ovaries of Boer goats; this finding is not consistent with the expression level in the estrous ovaries of uniparous and multiparous AWGs. Possibly, this result may have been because the tested ewes were at different stages of the estrous cycle or because of species differences. This study is the first to show a relationship between FER1L4 and SRD5A2 genes and kidding numbers.

\section{Tissue specific expression of FER1L4 and SRD5A2}

The expression of FER1L4 and SRD5A2 showed obvious tissue specificity in both AWGs and Boer goats. FER1L4 was expressed in the ovaries of AWGs and Boer goats, but showed little evidence of expression in heart, kidney, and uterus. SRD5A2 was expressed in the ovaries of AWGs and Boer goats, but showed little evidence of expression in the heart, lung, uterus, and small intestine; it also showed specifically high expression in the liver of uniparous AWGs and in Boer goats.

The above results indicate that FER1L4 and SRD5A2 genes were both expressed in the ovaries of AWGs and Boer goats, and highly expressed in the liver, suggesting they may also be relevant to goat fecundity. Our observations also indicate their function may not be limited to specific breeds, and that they may also participate in a variety of other physiological activities such as digestion and detoxification.

\section{CONCLUSION}

On the basis of screening for differential gene expression in the ovaries of uniparous and multiparous AWGs, we identified FER1L4 and SRD5A2 genes as the two genes showing highest differential expression between uniparous and multiparous AWGs. Our preliminary analysis examined the relationship between these genes and the high fertility in goats. This analysis indicated that both genes may be associated with high fecundity in AWG, but their relationship to fertility in Boer goats is not clear. Further research will be necessary to elucidate this relationship.

\section{Conflicts of interest}

The authors declare no conflict of interest.

\section{ACKNOWLEDGMENTS}

Research supported by the National Natural Science Foundation of China (\#31301934 and \#31372310), the Agricultural Science and Technology Innovation Program of China (\#ASTIPIAS13) and the Livestock and Poultry Sharing Platform in China.

\section{REFERENCES}

Akalu A, DImajian DA, Highshaw RA, Nichols PW, et al. (1999). Somatic mutations at the SRD5A2 locus encoding prostatic steroid 5-alpha-reductase during prostate cancer progression. J. Urol. 161: 1355-1358.

Benjamini Y, Drai D, Elmer G, Kafkafi N, et al. (2001). Controlling the false discovery rate in behavior genetics research. Behav. Brain Res. 125: 279-284.

Davis GH, Galloway SM, Ross IK, Gregan SM, et al. (2002). DNA tests in prolific sheep from eight countries provide new evidence on origin of the Booroola (FecB) mutation. Biol. Reprod. 66: 1869-1874. 
Dong Y, Xie M, Jiang Y, Xiao N, et al. (2013). Sequencing and automated whole-genome optical mapping of the genome of a domestic goat (Capra hircus). Nat. Biotechnol. 31: 135-141.

Ellsworth K and Harris G (1995). Expression of the 1 and 2 steroid 5 alpha-reductase in human fetal tissues. Biochem. Biophys. Res. Commun. 215: 774-780.

Galloway SM, McNatty KP, Cambridge LM, Laitinen MP, et al. (2000). Mutations in an oocyte-derived growth factor gene (BMP15) cause increased ovulation rate and infertility in a dosage-sensitive manner. Nat. Genet. 25: 279-283.

Gao P, Chen AL, Zhao QL, Shen XJ, et al. (2013). Differentially expressed genes in the ovary of the sixth day of pupal "Ming" lethal egg mutant of silkworm, Bombyx mori. Gene 527: 161-166.

Geng RQ, Yuan C and Chen YL (2013). Exploring differentially expressed genes by RNA-Seq in cashmere goat, Capra hircus: skin during hair follicle development and cycling. PLoS One 8: e62704.

Goodarzi MO, Shah NA, Antoine HJ, Pall M, et al. (2006). Variants in the 5- alpha-reductase type 1 and type 2 genes are associated with polycystic ovary syndrome and the severity of hirsutism in affected women. J. Clin. Endocrinol. Metab. 91: 4085-4091.

Gunawan A, Sahadevan S, Neuhoff C, Große-Brinkhaus C, et al. (2013). RNA deep sequencing reveals novel candidate genes and polymorphisms in boar testis and liver tissues with divergent androstenone levels. PLoS One 8: e63259.

Hackett NR, Butler MW, Shaykhiev R, Salit J, et al. (2012). RNA-Seq quantification of the human small airway epithelium transcriptome. BMC Genomics 13: 82.

Hanrahan JP, Gregan SM, Mulsant P, Mullen M, et al. (2004). Mutations in the genes for oocyte-derived growth factors GDF9 and BMP15 are associated with both increased ovulation rate and sterility in Cambridge and Belclare sheep, (Ovis aries). Biol. Reprod. 70: 900-909.

Hochberg Z, Chayen R, Reiss N, Falik Z, et al. (1996). Clinical, biochemical, and genetic findings in a large pedigree of male and female patients with steroid 5-alpha-reductase 2 deficiency. J. Clin. Endocrinol. Metab. 81: 2821-2827.

Huang W and Khatib H (2010). Comparison of transcriptomic landscapes of bovine embryos using RNA-Seq. BMC Genomics 11: 711.

Ishida-Takagishi M, Enomoto A, Asai N, Ushida K, et al. (2012). The Dishevelled-associating protein Daple controls the noncanonical Wnt/Rac pathway and cell motility. Nat. Commun. 3: 859.

Kang HJ, Imperato-McGinley J, Zhu YS and Rosenwaks Z (2014). The effect of 5a-reductase-2 deficiency on human fertility. Fertil. Steril. 101: 310-316.

Kaefer M, Audia JE, Bruchovsky N, Goode RL, et al. (1996). Characterization of type I 5-alpha-reductase activity in DU145 human prostatic adenocarcinoma cells. J. Steroid. Biochem Mol. Biol. 58: 195-205.

Kostyrko A, Antkowiak J, Warenik-Szymankiewiego A and Trzeciak WH (1994). The importance of DNA analysis in the diagnosis of steroid 5-alpha-reductase deficiency. Ginekol. Pol. 65: 400-407.

Li Q, Zhu Y, He J, Wang M, et al. (2013). Steroid 5-alpha-reductase type2, SRD5A2: V89L and A49T polymorphisms and sporadic prostate cancer risk: a meta-analysis. Mol. Biol. Rep. 40: 3597-3608.

Li R, Yu C, Li Y, Lam TW, et al. (2009). SOAP2: An improved ultrafast tool for short read alignment. Bioinformatics 25: 19661967.

Liu Z, Shao Y, Tan L, Shi H, et al. (2014). Clinical significance of the low expression of FER1L4 in gastric cancer patients. Tumor Biol. 35: 9613-9617

Kmieć M and Terman A (2006). Associations between the prolactin receptor gene polymorphism and reproductive traits of boars. J. Appl. Genet. 47: 139-141.

Mortazavi A, Williams BA, McCue K, Schaeffer L, et al. (2008). Mapping and quantifying mammalian transcriptomes by RNASeq. Nat. Methods 5: 621-628.

Mulsant P, Lecerf F, Fabre S, Schibler L, et al. (2001). Mutation in bone morphogenetic protein receptor-IB is associated with increased ovulation rate in Booroola Merino ewes. Proc. Natl. Acad. Sci. USA 98: 5104-5109.

Song H, Sun W, Ye G, Ding X, et al. (2013). Long non-coding RNA expression profile in human gastric cancer and its clinical significances. J. Transl. Med. 11: 225.

Sun W, Chang H, Musa HH and Chu MX (2010). Study on relationship between microsatellite polymorphism and producing ability on litter size trait of Hu sheep in China. Afr. J. Biotechnol. 9: 8704-8711.

Yokoi H, Tsuruo Y, Miyamoto T and Ishimura K (1998). Steroid 5 alpha-reductase type1 immunolocalized in the adrenal gland of normal, gonadectomized, and sex hormone-supplemented rats. Histochem. Cell Biol. 109: 127-134.

Zhang LP, Gan QF, Zhang XH, Li HD, et al. (2009). Detecting a deletion in the coding region of the bovine bone morphogenetic protein 15 gene (BMP15). J. Appl. Genet. 50: 145-148. 